\title{
ESTUDO DA VIABILIDADE DE TRATAMENTO FÍSICO PARA ÁGUA DE RESFRIAMENTO
}

\author{
R. SCHALY ${ }^{1}$, É. BRESCIANI $^{1}$ e R. GUARDANI ${ }^{1}$ \\ ${ }^{1}$ Universidade São Paulo, Departamento de Engenharia Química \\ E-mail para contato: rjcamara@terra.com.br
}

\begin{abstract}
RESUMO - O objetivo deste estudo é avaliar a viabilidade técnico-econômica da implantação do tratamento eletromagnético, para água de resfriamento, substituindo ou complementando o tratamento químico convencional. $\mathrm{O}$ estudo apresenta o princípio de funcionamento do processo físico de tratamento de água baseado na aplicação de campo eletromagnético e é focado na redução dos custos decorrentes da menor adição de produtos químicos, redução na vazão de purga e de reposição de água, redução na frequência de limpeza dos equipamentos e redução da carga poluente nos efluentes líquidos. Os resultados são aplicados a uma torre de resfriamento industrial para a qual foi elaborado um modelo matemático considerando as taxas de consumo e especificações de água de resfriamento, parâmetros do processo de tratamento de água convencional e eletromagnético. São apresentados resultados de estudo de sensibilidade para diferentes cenários, em que são identificados os fatores com maior efeito sobre os custos.
\end{abstract}

\section{INTRODUÇÃO}

\subsection{A disponibilidade da água}

Segundo a UNESCO (2012), 70\% da água doce destinam-se à irrigação das lavouras, $20 \%$ são usadas pela indústria e $10 \%$ destinam-se aos diversos usos domésticos. O percentual utilizado pela indústria varia conforme a renda do país. Países de baixa renda utilizam cerca de $5 \%$ na indústria e países de alta renda podem consumir até $40 \%$. No contexto industrial ainda, a captação que é feita tanto das fontes subterrâneas quanto das superficiais é muito maior do que o consumido.

As plantas industriais utilizam água nos processos para reposição em torres de resfriamento, geração de vapor, processos (retificação, atomização, inertização, selagem, granulação de escória ou metais etc.), matéria-prima (bebidas, alimentos, etc.), combate a incêndio, serviços gerais, consumo das pessoas (cozinha, banheiros, etc.)

Dentre os consumos acima, para grande parte das plantas, a utilização da água para a reposição das torres de resfriamento representa o maior consumidor. No caso de refinarias de petróleo, o consumo total de água é da ordem de $1 \mathrm{~m} 3$ por $\mathrm{m} 3$ de petróleo refinado e a água para reposição de torres de resfriamento representa de 40 a $50 \%$ do consumo total. No caso de petroquímicas, o consumo de água atinge cerca de $5 \mathrm{~m}^{3}$ por $\mathrm{m}^{3}$ de nafta processada e a água usada em torres de resfriamento também atinge cerca de 50\% do total consumido, BRESCIANI et al. (2006). 
A indústria cada vez mais busca trabalhar em circuitos fechados de água para evitar o desperdício e a captação de água fresca. Nesse sentido, o tratamento magnético pode reduzir a quantidade de material depositado em equipamentos, diminuindo a resistência térmica que afeta a eficiência da troca de calor e reduzindo, dessa forma, a necessidade de aumentar a vazão de circulação de água de resfriamento e consequentemente a sua reposição e descarte.

\subsection{Aplicações do campo eletromagnético no tratamento de água}

Nos últimos anos, o tratamento magnético da água vem sendo estudado principalmente para o combate da incrustação e como bactericida.

Os trabalhos científicos publicados, KOCKMARSKY, 1996; PARSONS et al., 1997; WANG et al., 1997; GABRIELLI et al., 2001; FATHIA et al., 2010; mostram o efeito de redução na formação de incrustação na água. Afirmam também que os mecanismos de ação do campo magnético sobre a água ainda não são totalmente compreendidos.

Para o pesquisador e inventor PANDOLFO (2003), quando o campo magnético é aplicado na água carbonatada, há a formação de carbonato de cálcio na forma de pequenos cristais, como se fossem pequenas sementes que vão crescendo ao longo do tempo no interior do líquido. Esses pequenos grãos dão origem ao cristal na forma de aragonita que é mais mole do que a calcita. A forma de cristal calcita é a que se adere à superfície dos equipamentos. Sem a aplicação do campo magnético, haveria a preferência de ocorrer o precipitado na forma de calcita que é a cristalização considerada aderente.

No equipamento apresentado pelo pesquisador PANDOLFO (2003) há dois efeitos associados, sendo um deles o campo magnético gerado pelas solenóides com um valor de fluxo magnético máximo de $0,02 \mathrm{~T}$ e uma onda pulsante com uma frequência da ordem de 1,15MHz. Esse equipamento dificulta a formação da incrustação e pode ser resultante da combinação do campo com a onda pulsante.

Nos trocadores de calor, um fluxo turbulento plenamente desenvolvido apresenta velocidade quase nula próxima às paredes das tubulações. A aragonita é a forma do cristal predominante na temperatura acima de $80^{\circ} \mathrm{C}$; no entanto, conforme OGINO et al. (1987), após 1000 minutos converte-se totalmente em calcita. Em torres de resfriamento de grande porte, o tempo de residência médio pode chegar a 600minutos ou mais. Já nas paredes esse tempo de residência é muito maior, pois a velocidade é praticamente nula e há tempo suficiente para que ocorra a transformação de aragonita em calcita que é a forma mais dura de cristalização. Com base nas afirmações dos trabalhos de CHO et al. (2005) e de PANDOLFO (2003) é possível dizer que o tratamento magnético favorece a precipitação do carbonato de cálcio na fase bulk, diminuindo a disponibilidade dos íons para formar a incrustação nas paredes das tubulações. O cristal formado no interior do líquido acaba sendo drenado sem que haja a formação da incrustação. 


\section{METODOLOGIA PARA ESTUDO DA VIABILIDADE TÉCNICO- ECONÔMICA DO PROCESSO}

Esse trabalho se propõe a construir um modelo matemático para o estudo da viabilidade técnicoeconômica da utilização do tratamento magnético em um circuito de água de resfriamento. Essa avaliação será conduzida considerando a comparação dos custos totais entre as situações (1) com tratamento convencional e (2) com tratamento híbrido onde a aplicação de campo magnético possibilita a redução do uso de produtos químicos e de água de reposição.

\subsection{Modelagem dos custos para o tratamento convencional}

Custo de Investimento - $\operatorname{cinv}(1)$ : O custo anual de investimento (em US\$/ano)

Custos operacionais - y(1) a y(11): O custo operacional é resultante da soma das parcelas y(1) a $\mathrm{y}(11)$, sendo que várias delas são dependentes da vazão Wr que é função da quantidade de água evaporada(We) em $\mathrm{m}^{3} / \mathrm{h}$ e deo ciclo de concentração Ccn.

Custo anual da água de reposição - y(1): O custo da água de reposição é dado pelo custo (Xwr) do $\mathrm{m}^{3}$ da água de reposição multiplicado pela vazão de reposição.

$$
y(1)=X w r * W r * n \circ \frac{\text { horas }}{\text { ano }} \therefore y(1)=X w r * W e\left(\frac{C c n}{C c n-1}\right) * n \frac{\text { horas }}{\text { ano }}
$$

Custos anuais dos produtos químicos $-\mathrm{y}(2), \mathrm{y}(3), \mathrm{y}(4)$ : PQ1, PQ2, PQ3 = Quantidade de produto químico anticorrosivo, anti-incrustante e antibactericida, utilizada $\mathrm{em} \mathrm{kg} / \mathrm{m}^{3}$ de água de reposição e $\mathrm{Xpq} 1, \mathrm{Xpq} 2, \mathrm{Xpq} 3$ = custo dos produto em US\$/kg.

$$
\begin{aligned}
& y(2)=P Q 1 * X p q 1 * W e\left(\frac{C c n}{C c n-1}\right) * n{ }^{\circ} \text { horas /ano } \\
& y(3)=P Q 2 * X p q 2 * W e\left(\frac{C c n}{C c n-1}\right) * n{ }^{\circ} \text { horas } / \text { ano } \\
& y(4)=P Q 3 * X p q 3 * W e\left(\frac{C c n}{C c n-1}\right) * n{ }^{\circ} \text { horas } / \text { ano }
\end{aligned}
$$

Custo anual do tratamento de efluente - y(5): Definido como 0,5 vezes o custo do $\mathrm{m}^{3}$ da água de reposição (Xwr). O custo do tratamento de efluentes poderá ser relacionado conforme a equação a seguir, na qual Wd é a quantidade de água de descarte e Wc água de recirculação em $\mathrm{m} / \mathrm{h}$, fA é o fator de arraste:

$$
\begin{aligned}
y(5)=0,5 * X w r * W d * n \circ \frac{\text { horas }}{\text { ano }} \therefore y(5) \\
=0,5 * X w r *\left[\frac{W e}{C c n-1}-f A * W c\right] * n \frac{\text { horas }}{\text { ano }}
\end{aligned}
$$

Custo anual de manutenção da torre $-\mathrm{y}(6)$ 
Custo anual da perda de energia decorrente da formação de incrustação - y(7): A equação E.6 que representa a relação do fouling com o tempo em termos do valor assintótico $R f^{*}$.

$$
R f=R f^{*}\left[1-e^{-\left(\frac{t}{t c}\right)}\right]
$$

Na equação E.6, tc é definido como o tempo em que o fouling atinge $63 \%$ do valor assintótico, pois para $\mathrm{t}=\mathrm{tc}$, tem-se: $R f=R f^{*}\left[1-e^{-(1)}\right]$ e $R f \sim R f^{*} x 0,63$, dessa forma pode-se admitir que tc $0,63 *$ t*.A resistência "fouling" é dada pela seguinte equação, conforme AWAD (2011):

$$
R f=\frac{1}{U f}-\frac{1}{U c}
$$

Uf é o coeficiente global de troca térmica para o tubo que já sofreu "fouling" e Uc é o coeficiente global de troca térmica do tubo limpo. Substituindo-se a relação de Rf da equação E.6 em E.7, obtém a relação pra Uf em função da resistência fouling estimada Rf* e do tempo de operação.

$$
U f=1 / \frac{1}{U c}+R f^{*}\left[1-e^{-\left(\frac{t}{t c}\right)}\right]
$$

A fim de manter a mesma condição de troca térmica, a vazão de água de resfriamento deverá ser aumentada em função do fouling formado. A quantidade de água de circulação em função do calor trocado é apresentada na equação E.8 da seguinte forma:

$$
Q c=W c . C p . \Delta T e Q f=W f . C p . \Delta T \therefore \frac{Q f}{Q c}=\frac{W f}{W c} \therefore W f=W c * \frac{Q f}{Q c}
$$

A diferença de vazão entre um trocador limpo e um trocador sujo $\Delta W$ é a diferença entre Wc e Wf que pode ser escrita como uma função da resistência térmica, através da combinação entre as equações E.8 e E.9, resultando na equação E.10.

$$
\begin{gathered}
\Delta W=W c-W f \therefore \Delta W=W c-W c * \frac{Q f}{Q c} \therefore \Delta W=W c\left(1-\frac{Q f}{Q c}\right) \\
\therefore \Delta W=W c\left(1-\frac{1}{U c * R f+1}\right) \therefore \Delta W=W c\left(\frac{U c * R f}{U c * R f+1}\right)
\end{gathered}
$$

Para o cálculo da potência requerida no bombeamento foram usadas as relações de variação de pressão, $\Delta p$, e da energia requerida pela bomba PW, conforme INCROPERA et. al (2006), na qual:

$$
P W=\Delta p * W c / 3600
$$

$\Delta p$ em $N / m^{2}$ e Wc em $\mathrm{m}^{3} / \mathrm{h}$.

Através da equação E.11 pode-se deduzir a expressão para a potência requerida em um trocador sujo. Para o trocador de calor sujo haverá um aumento na potencia em função do aumento de vazão dado pela soma de $\mathrm{Wc}^{\Delta}{ }^{\Delta} \mathrm{W}$. Em um processo sem incrustação, a potência é definida pela equação E.11 e num processo em que há incrustação a perda de energia será dada por $\Delta W$ e será representada pela 
equação E.12 conforme segue:

$$
P W=\Delta p * \frac{\Delta W}{3600} \therefore P W=\Delta p * W c\left(\frac{U c * R f}{U c * R f+1}\right) / 3600
$$

$\mathrm{y}(7)=\mathrm{n}^{\circ}$ horas/ano*PW $* \mathrm{XW} / \mathrm{Ef}$, na qual o XW é o custo do Watt em US\$/W*h e Ef é eficiência do bombeamento que pode ser definido como 0,80. Em resumo:

$$
y(7)=\Delta p * \frac{W c\left(\frac{U c * R f}{U c * R f+1}\right)}{3600} * \frac{X W}{0,80} * \frac{n \text { horas }}{\text { ano }}
$$

Custo anual de energia elétrica para a operação - y(8) : O custo de energia para suprir bombas e ventiladores das torres é considerado o mesmo nos dois casos conforme apresentado na equação E.14.

$$
y(8)=P W * X W * \frac{n \text { horas }}{\text { ano }}
$$

Custo anual de perda de produção - y(9)

Custo anual da manutenção dos equipamentos consumidores de água y(10)

Em resumo, ct1 pode ser representado pela equação E.17, substituindo o termo $n^{\circ}$ horas/ano por t/ano e a expressão de Rf pela expressão da variação do fouling no tempo, tem-se:

$$
\begin{gathered}
c t 1=\operatorname{cinv} 1+X w r \cdot\left[W e \cdot\left(\frac{C c n}{C c n-1}\right)\right] \cdot \frac{t}{a n o}+\sum_{i=1}^{3} P Q i \cdot X p q i \cdot\left[W e \cdot\left(\frac{C c n}{C c n-1}\right)\right] \cdot \frac{t}{a n o}+ \\
0,5 . X w r \cdot\left[\frac{W e}{C c n-1}-f A \cdot W c\right] \cdot \frac{t}{a n o}+y(6)+\Delta p \cdot W c\left[\frac{U c \cdot R f^{*}\left[1-e^{-\left(\frac{t}{t c}\right)}\right]}{U c \cdot R f^{*}\left[1-e^{-\left(\frac{t}{t c}\right)}\right]+1}\right] \cdot \frac{1}{3600} \cdot \frac{X W}{0,80} \cdot \frac{t}{a n o} \\
+P W \cdot X W \cdot \frac{t}{a n o}+y(9)+y(10)+y(11)
\end{gathered}
$$

\subsection{Modelagem dos custos para o tratamento hibrido}

Para o sistema hibrido, equação E.16, é necessário considerar o custo dos equipamentos magnéticos, $\mathrm{cEq}(1), \mathrm{cEq}(2), \mathrm{cEq}(3)$ e o fator de redução de utilização de produto químico, $\mathrm{RFM}(1)$, RFM(2) e RFM(3). Além disso, para o fator de redução de incrustação devido ao tratamento magnético, considerou-se o fator RFM(4), valor baseado no trabalho de CHO et al. (2005). Todos esses fatores variam de 0 a 1 .

$$
\begin{aligned}
& c t 2=\operatorname{cinv} 1+c E q 1+c E q 2+c E q 3+X w r\left[W e \cdot\left(\frac{C c n 1}{C c n 1-1}\right)\right] \cdot \frac{t}{a n o}+ \\
& \sum_{i=1}^{3} P Q i . X p q i \cdot\left[W e \cdot\left(\frac{C c n 1}{\operatorname{ccn} 1-1}\right)\right] \cdot R F i \cdot \frac{t}{a n o}+0,5 \cdot X w r \cdot\left[\frac{W e}{c c n 1-1}-f A \cdot W c\right] \cdot \frac{t}{a n o}+z(6)+
\end{aligned}
$$


$\Delta p \cdot \frac{W c}{3600} \cdot\left[\frac{U c . R F M 4 . R f^{*}\left[1-e^{-\left(\frac{t}{t c}\right)}\right]}{U c . R F M 4 . R f^{*}\left[1-e^{-\left(\frac{t}{t c}\right)}\right]+1}\right] \cdot \frac{X W}{0,80} \cdot \frac{t}{\text { ano }}+y 8+X W \cdot(P W 2+P W 3+P W 4) \cdot \frac{t}{\text { ano }}+z(9)+z(10)+$ $y(11)$

Em uma primeira análise, pode-se dizer que a função objetivo poderá ser dada por:

$$
\text { min } S=C t 2-C t 1
$$

Sujeito à: $c t 2-c t 1<0, z(6)=y(6) ; z(11)=y(11) ; z(2)=0$, quando $0 \geq R F M \leq 1,0 \geq t \leq$ parada para manutenção definida em horas

\section{APLICAÇÃO EM UM CASO INDUSTRIAL}

Foi considerado um estudo de caso para uma torre de resfriamento industrial, operando com uma vazão de circulação $\mathrm{Wc}$ de $12500 \mathrm{~m}^{3} / \mathrm{h}$ e com tratamento químico para combate a corrosão, incrustação e crescimento microbiológico. Os parâmetros de quantidades de produto e custo dos produtos foram extraídos da indústria para a qual a análise foi realizada. Considerando uma velocidade de $2 \mathrm{~m} / \mathrm{s}$ para o escoamento da água de resfriamento, o volume da bacia de resfriamento de $360 \mathrm{~m}^{3}$ e uma vazão de reposição máxima e adotando um ciclo de concentração 6 (considerando a concentração do carbonato de cálcio) foi possível determinar a quantidade de equipamentos necessários para o tratamento físico da água, conforme configuração apresentada na figura 1 . O diâmetro dos equipamentos e a potência dos mesmos foram extraídos do catálogo do fabricante, SONICAL, 2012, do tipo de dispositivo magnético escolhido, o qual utiliza um arranjo especial de solenoides para maximizar o campo eletromagnético na água que passa pelo centro do mesmo.

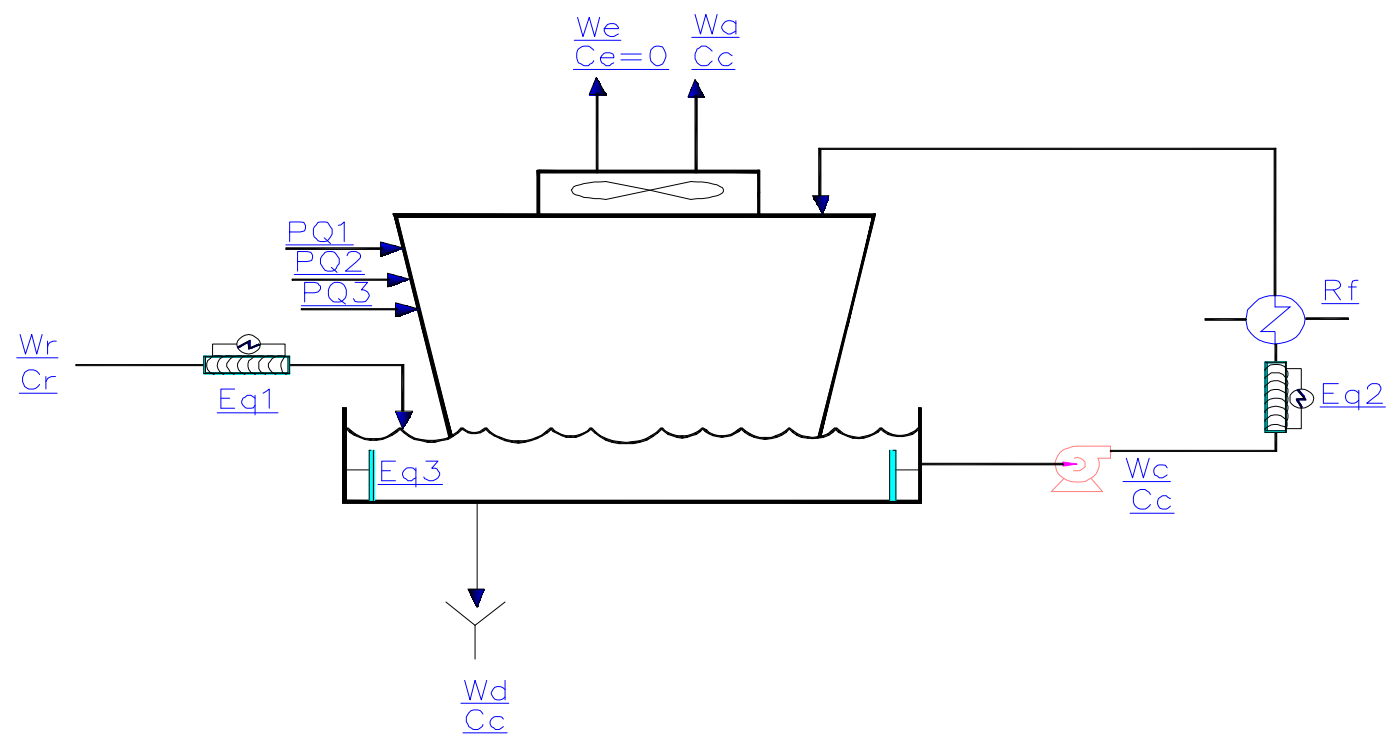

Figura 1: Representação do sistema de resfriamento. Eq1 = 1, Eq2 = 14 e EQ3 = 7 . 


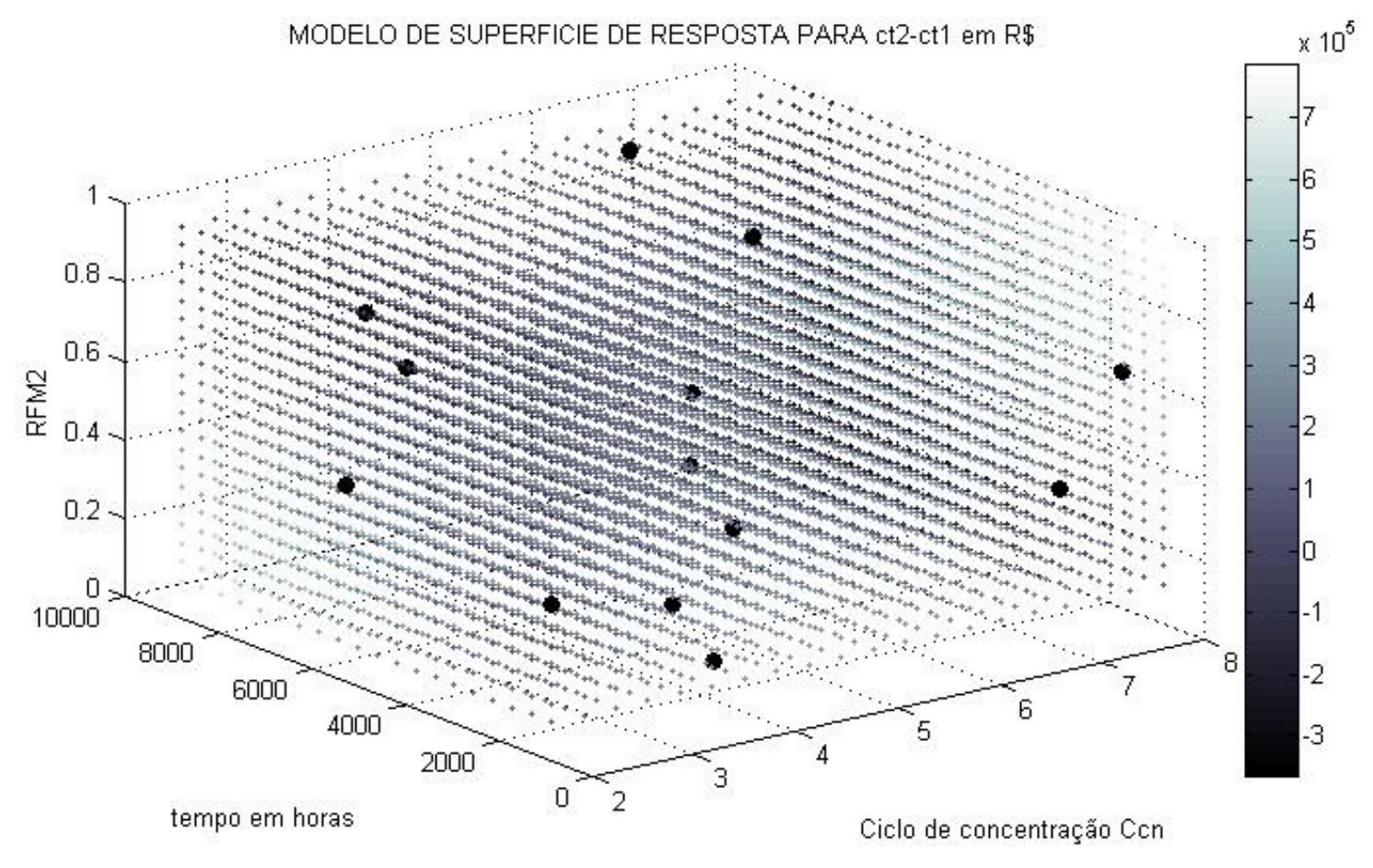

Figura 2: Análise da superfície de resposta considerando três anos de operação com o tratamento hibrido.

\section{CONCLUSÕES}

A figura 2 apresenta o resultado da diferença de ct2-ct1 em R $\$$ considerando a variação do ciclo de concentração para o carbonato de cálcio e a utilização do produto químico anti-incrustante. $O$ fator de utilização de produto anti-incrustante tem um valor negativo para a diferença ct2-ct1 quando o RFM2 é igual a 0,3 , o que representa que a redução é de $70 \%$ da utilização do produto químico. $\mathrm{O}$ ciclo de concentração para esse caso pode ser de 3 a 7 para que o valor da diferença seja negativo representado pela cor mais escura. Foi considerada para essa análise uma depreciação de $30 \%$ do capital investido nos equipamentos magnéticos para que o processo fosse economicamente viável. O custo de investimento nos equipamentos físicos é um fator limitante para a implantação do tratamento magnético num período inferior a 3 anos. Para a geração desses resultados, considerou-se não utilizar produto para combate a corrosão, visto que o $\mathrm{pH}$ recomendado para a utilização desses equipamentos é acima de 8 e considerouse também não utilizar produto bactericida, o qual seria substituído pelas placas de eletrodos (Eq3).

Na figura 1 há a indicação de quantos equipamentos são necessários para tratar a água de reposição $(1 \mathrm{Eq} 1)$, para tratar a água de circulação $(\mathrm{Eq} 2=14)$ e para tratar a água da bacia de resfriamento (Eq3=7). O custo maior de investimento está associado ao Eq2, pois a vazão de circulação é muito elevada nessa torre. De acordo com esse resultado e premissas adotadas, a viabilidade do tratamento é interessante, desde que se considere um tempo de pagamento do investimento de pelo menos 3 anos para o caso estudado. 


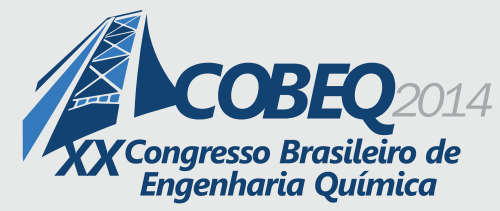

\section{REFERÊNCIAS}

AWAD, M. M. Fouling of heat transfer surfaces, heat transfer - theoretical Analysis, experimental investigations and industrial systems, Prof. Aziz Belmiloudi (Ed.) ISBN: 978-953-307-226-5, Intech,2011, disponível em:

http://www.intechopen.com/books/heat-transfer-theoretical-analysis-experimental-investigations-andindustrial-systems/fouling-of-heat-transfer-surfaces, acesso em 28/05/2013.

BRESCIANI, A. E.; ALVES, R. M. B.; Guardani, R.; Nascimento, C. A. O. Água nas Indústrias: Otimização do Uso e Reuso. Revista Brasileira de Engenharia Química, v. 12, p. 18-23, 2006.

CHO, Y. I., LANE, J.; KIM W. Pulsed-power treatment for physical water treatment. International Communications in Heat and Mass Transfer, vol. 32, p. 861-871, 2005.

FATHIA, A; MOHAMED, T.; CLAUDE, G.; MAURIN, G.; MOHAMED, B. A. Effect of a magnetic water treatment on homogeneous and heterogeneous precipitation of calcium carbonate. Water Research. Vol. 40 (10), p. 1941-1950, 2006.

GABRIELLI, C.; JAOUHARI, R.; MAURIN, G.; KEDDAM, M. Magnetic Water Treatment for Scale Prevention. Water Research. Vol. 35, No. 13, p. 3249-3259, 2001.

INCROPERA, F. P.; DEWITT, D. P.; BERGMAN, T. L.; LAVINE, A. S.Fundamentals of heat end mass transfer, 6th edition, p. 497-498, March, 2006.

KOCHMARSKY, V. Magnetic treatment of water: possible mechanisms and conditions for aplications. Mag. Electrical Sep., vol.7, p. 77-107, 1996.

OGINO, T.; SUZUKI, T.; SAWADA, K. The formation and transformation mechanism of calcium carbonate in water. Geochim. Cosmochim. Acta, vol. 51, p. 2757-2767 (1987).

PANDOLFO, M. Variable Resonance Descaling Descalcifier Device Connected to a Forced Sequential Rephasing Transformer. United States Patent US 6506299B1, 14/01/2003.

PARSONS, S. A.; WANG, B. L.; JUDD, S. J.; STEPHENSON, T. Magnetic treatment of calcium carbonate scale-effect of pH control. Water Research, vol. 31 (2), p. 339-342, 1997.

SONICAL: Catalogo Prodotti Sonical, 2012

UNESCO (United Nations Educational, Scientific and Cultural Organization) disponível em: http://unesdoc.unesco.org/images/0021/002171/217175E.pdf.

Acessoem: 07/11/2012.

WANG, Y.; BABCHIN, A. J.; CHERNYI, L. T.; CHOW, R. S.; SAWATZKY, R. P. Rapid Onset of Calcium Carbonate Crystallization Under the Influence of a Magnetic Field, Wat. Res. Vol. 31, No. 2, p. 346-350, 1997. 